\title{
A PROSPECTIVE STUDY ON TOTAL KNEE ARTHROPLASTY OUTCOMES IN NORTH OF IRAN
}

\author{
Abdolmajid Gharajeh ${ }^{1}$, Nasser Janmohammadi², Seyed Mokhtar Esmaeilnejad-Ganji ${ }^{3}$ \\ 1 Postgraduate Resident, Student Research Committee, Babol University of Medical Sciences, Babol, Iran. \\ 2 Professor, Mobility Impairment Research Centre, Health Research Institute, Babol University of Medical Sciences, Babol, Iran. \\ ${ }^{3}$ Associate Professor, Department of Orthopaedics, Shahid Beheshti Hospital, School of Medicine, Babol University of Medical Sciences, \\ Babol, Iran.
}

\section{BACKGROUND}

ABSTRACT

Total knee arthroplasty is one of the suitable procedures to treat the patients with severe knee arthritis. The aim of this study was to evaluate the outcomes of total knee arthroplasty in Babol, northern Iran, for the first time.

\section{MATERIALS AND METHODS}

In this prospective observational study, all patients who underwent total knee arthroplasty in Rohani and Shahid Beheshti Teaching Hospitals during 2015-2017 were enrolled. Subjects with previous knee replacement, contraindication for major surgeries and failure in follow-up were excluded. To assess the surgery outcomes, we used the Knee Society Score which was categorised by functional score and knee score. Patients were followed up for eighteen months after the operation. The pre- and post-operative scores were compared by SPSS using paired t-test analysis.

\section{RESULTS}

Of 70 patients, $15(21.4 \%)$ were male and others were female. The most common cause of total knee arthroplasty was osteoarthritis $(n=53,75.7 \%)$. Over the six-month follow-up, the patients' functional score significantly increased from $32.4 \pm 30.95$ to $66.9 \pm 61.31(\mathrm{p}<0.001)$. The knee score also had a significant increase from $29.4 \pm 74.12$ to $83.6 \pm 43.02(\mathrm{p}<0.001)$. The functional score significantly improved at eighteen months of surgery compared with that measured at six months of surgery ( $p=0.044)$. Thirty-one patients (44.3\%) had excellent status based on knee scoring system, 27 patients (38.6\%) had good status, 10 patients $(14.3 \%)$ had fair status and 2 patients $(2.8 \%)$ had weak status at the final follow-up.

\section{CONCLUSION}

The present study showed that total knee arthroplasty could acceptably improve knee function in patients with knee arthropathy who underwent the surgery, and this can be correlated with their satisfaction.

\section{KEY WORDS}

Knee Joint, Arthroplasty, Replacement.

HOW TO CITE THIS ARTICLE: Gharajeh A, Janmohammadi N, Ganji SME. A prospective study on total knee arthroplasty outcomes in North of Iran. J. Evolution Med. Dent. Sci. 2018;7(47):5084-5088, DOI: 10.14260/jemds/2018/1130

\section{BACKGROUND}

Total knee arthroplasty is one of the methods of treatment in the patients with end-stage knee arthritis and is one of the most successful reconstruction operations in orthopaedics.(1,2) In the early 1970s along with development of condylar prosthesis of knee, modern arthroplasty started. Over the past years, total knee arthroplasty has been increasingly used in the world, especially in the developed countries. In the United States, more than 650,000 surgeries were recorded in 2008 with a seven-fold increase from 19711976.(3,4) This frequency was more than 77,500 in the United Kingdom in 2009.(5)

The indication for total knee arthroplasty is mainly the severe pain caused by severe arthritis with or without deformity.(6-8) The decision to operate is affected by patient's

'Financial or Other Competing Interest': Dr. Esmaeilnejad-Ganji reports Grants from Vice chancellor for the Research, outside the submitted work.

Submission 09-10-2018, Peer Review 02-11-2018,

Acceptance 09-11-2018, Published 19-11-2018.

Corresponding Author:

Dr. Seyed Mokhtar Esmaeilnejad-Ganji,

Department of Orthopaedics,

Babol University of Medical Sciences,

Ganjafrooz Street, Babol, Mazandaran, Iran.

E-mail: smsnganji20@gmail.com

DOI: $10.14260 /$ jemds $/ 2018 / 1130$ symptoms (e.g. Severe refractory knee pain, substantial disability, no response to non-operative treatments).(9)

In most cases undergoing total knee arthroplasty, patients are satisfied by functional recovery and reduced pain. It was reported that total knee arthroplasty improves health-related quality of life in about $90 \%$ of the patients. However, this surgery is potentially associated with a series of complications such as infection, implant loosening, thromboembolic disease, implant fracture and readmission.(10,11)

Although, there are different studies evaluating the outcomes of total knee arthroplasty in the world, this topic has not been assessed in our region, Babol, northern Iran, yet and we do not have any data to see if total knee arthroplasty is associated with acceptable outcomes or not. Therefore, this study was performed with the aim of evaluating the outcomes of total knee arthroplasty in Babol. These findings would help the Iranian orthopaedic surgeons to make a better decision when facing complicated patients.

\section{MATERIALS AND METHODS Study Population}

In this prospective observational study, all patients who underwent total knee arthroplasty between January 2015 and January 2017 were included. The surgeries were performed in Shahid Beheshti and Ayatollah Rohani Teaching Hospitals. The exclusion criteria were as follows: 1) Previous 
knee replacement; 2) Contraindication for major surgeries; 3) Failure in follow-up.

\section{Data Collection}

The necessary data were obtained by checklist. The required information included demographic (Age, sex, occupation, educational level, marital status, body mass index), duration of surgery and post-operative complications. To assess knee function, we used the Knee Society Score. This clinical scoring system was initially released by the Knee Society in 1989.(12) The advantage of this system is that the patient's functional score is separate from the knee score. The functional score rates the patient's ability to walk a distance (50 scores) and climb stairs (50 scores). Use of assistive devices like cane or crutch will detract from the scores. The knee score is divided into the three following parts: 1) 50 scores for pain; 2) 25 scores for range of motion; 3) 25 scores for joint stability. Joint stiffness in bending, inability to open knee and poor knee alignment have negative scores in knee assessment. Totally, scores are rated excellent (80-100), good (70-79), fair $(60-69)$ and poor $(<60) .(13-15)$ In our study, the Knee Society Score was evaluated before the operation, six and eighteen months after the operation.

\section{Preoperative Protocol}

The aims of the study and plans for follow-up were clearly declared to the patients. The indication for total knee arthroplasty was determined by the orthopaedic surgeon. Knee x-ray image (anterior-posterior, lateral views) and long leg x-ray (alignment view) were taken for all subjects.

\section{Procedure of Surgery}

Spinal anaesthesia was induced in the patients by epidural administration of $2 \%$ lidocaine. A pneumatic tourniquet was used to produce a bloodless field. All surgeries were conducted by the same senior attending surgeon, through anterior approach and medial parapatellar capsulotomy. The procedures last 45-120 minutes. The implants used for total knee arthroplasty included femoral and tibial components and polyethylene insert. All knees got the same prosthesis.

\section{Postoperative Protocol}

Antibiotic was administered for the patients (Cefazolin $1 \mathrm{~g}$ intravenously every 6 hours) until the first 48 hours of surgery. For controlling the postoperative pain, we administered acetaminophen every 12 hours for maximum period of 2 weeks or piroxicam every 12 hours for maximum period of 1 week. After two days, range of motion exercises were performed as passive and active flexion and extension. These exercises were continued until strength of quadriceps muscle reached grade 5 (Normal strength) and knee flexion was greater than 100 degrees. All patients finally achieved more than 100 degrees of knee flexion.

\section{Statistical Analysis}

In order to data analysis, we used SPSS statistical software. The data underwent the descriptive analysis to provide the number, percentage and mean \pm standard deviation (SD). Moreover, paired t-test analysis was performed to compare the knee society score before and after total knee arthroplasty. A p-value $<0.05$ was considered statistically significant.

\section{Ethical Issues}

The study protocol was approved by the Ethical Committee of Babol University of Medical Sciences with code of MUBABOL.HRI.REC.1395.115. After explanation of the aim of study for the subjects, we collected the informed consent from them. The patients' information was kept confidential.

\section{RESULTS}

In this study 70 patients were finally assessed, of whom 15 $(21.4 \%)$ were male and $55(78.6 \%)$ were female. The mean age was $66.44 \pm 8.18$ years old, ranging from 52 to 80 years old. Other baseline information of the subjects are represented in Table 1.

Primary osteoarthritis was the most frequent cause of total knee arthroplasty in the subjects $(n=53,75.7 \%)$ followed by osteoarthritis secondary to trauma $(n=12$, $17.1 \%)$, multiple epiphyseal dysplasia $(n=3,4.3 \%)$ and osteosarcoma ( $\mathrm{n}=2,2.9 \%)$.

As indicated in Table 2, all parameters of the functional score including walking score and stair score significantly increased at six months of the surgery compared with before the surgery (both $\mathrm{p}<0.001$ ). The significant improvement in functional score was also seen at eighteen months of the operation compared with that at six months $(p=0.044)$. With respect to three parameters of the knee score, all significantly increased over the six months of total knee arthroplasty (Table 2). These parameters were non-significantly improved until eighteen months of the operation.

In total, fifty-eight patients (82.9\%) had excellent or good final knee score and $12(17.1 \%)$ had fair or poor score at the final follow-up (Figure 1). Figure 2 shows the outcome of total knee arthroplasty before and eighteen months after the surgery in a patient with severe osteoarthritis of right knee.

In relation to postoperative complications, 55 patients (78.6\%) had no any complications, whereas $7(10 \%)$ and 3 $(4.3 \%)$ patients suffered from infection and deep vein thrombosis, respectively. Rate of complications is shown in Table 3.

\begin{tabular}{|c|c|c|}
\hline Variable & Frequency & Percent \\
\hline \multicolumn{3}{|l|}{ Gender } \\
\hline Male & 15 & 21.4 \\
\hline Female & 55 & 78.6 \\
\hline \multicolumn{3}{|l|}{ Marital Status } \\
\hline Married & 11 & 15.7 \\
\hline Unmarried & 59 & 84.3 \\
\hline \multicolumn{3}{|l|}{ Occupational Status } \\
\hline Homemaker & 52 & 74.3 \\
\hline Self-employment & 13 & 18.6 \\
\hline Retired & 2 & 2.9 \\
\hline Employee & 3 & 4.3 \\
\hline \multicolumn{3}{|l|}{ Educational Level } \\
\hline Illiterate & 25 & 35.7 \\
\hline Lower diploma & 38 & 54.3 \\
\hline Diploma & 7 & 10 \\
\hline \multicolumn{3}{|l|}{$\begin{array}{c}\text { Body Mass Index } \\
\left(\mathrm{Kg} / \mathrm{M}^{2}\right)\end{array}$} \\
\hline$<18.5$ & 10 & 14.3 \\
\hline $18.5-24.9$ & 22 & 31.4 \\
\hline $25-29.9$ & 30 & 42.9 \\
\hline$\geq 30$ & 8 & 11.4 \\
\hline
\end{tabular}




\begin{tabular}{|c|c|c|c|c|c|}
\hline$\frac{0}{\frac{0}{2}}$ & 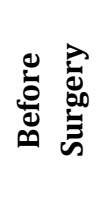 & 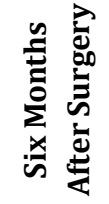 & $\frac{*}{\mathscr{*}}$ & 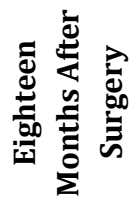 & 草 \\
\hline $\begin{array}{c}\text { Functional } \\
\text { score* }\end{array}$ & $\begin{array}{l}32.4 \pm \\
30.95\end{array}$ & $\begin{array}{l}66.9 \pm \\
61.31\end{array}$ & $<0.001$ & $\begin{array}{l}82.1 \pm \\
12.03\end{array}$ & 0.044 \\
\hline $\begin{array}{c}\text { Walking } \\
\text { score }\end{array}$ & $\begin{array}{l}18.1 \pm \\
29.97\end{array}$ & $\begin{array}{l}33.2 \pm \\
27.93\end{array}$ & 0.003 & $\begin{array}{l}40.3 \pm \\
39.17 \\
\end{array}$ & 0.219 \\
\hline Stair score & $\begin{array}{c}17.2 \pm \\
3.88\end{array}$ & $\begin{array}{l}42.1 \pm \\
24.99\end{array}$ & $<0.001$ & $\begin{array}{l}48.1 \pm \\
27.18\end{array}$ & 0.353 \\
\hline Knee score* & $\begin{array}{l}29.4 \pm \\
74.12\end{array}$ & $\begin{array}{l}83.6 \pm \\
43.02\end{array}$ & $<0.001$ & $\begin{array}{c}89.64 \pm \\
58.26\end{array}$ & 0.487 \\
\hline Pain score & $\begin{array}{c}1.0 \pm \\
0.2\end{array}$ & $\begin{array}{c}35.77 \pm \\
23.25\end{array}$ & $<0.001$ & $\begin{array}{l}42.5 \pm \\
58.66\end{array}$ & 0.374 \\
\hline $\begin{array}{c}\text { Range of } \\
\text { motion } \\
\text { score }\end{array}$ & $\begin{array}{l}8.2 \pm \\
5.09\end{array}$ & $\begin{array}{c}20.94 \pm \\
53.01\end{array}$ & 0.047 & $\begin{array}{l}22.9 \pm \\
34.77\end{array}$ & 0.796 \\
\hline $\begin{array}{c}\text { Stability } \\
\text { score }\end{array}$ & $\begin{array}{l}12.1 \pm \\
23.78\end{array}$ & $\begin{array}{l}19.5 \pm \\
16.30\end{array}$ & 0.034 & $\begin{array}{l}22.3 \pm \\
19.95\end{array}$ & 0.365 \\
\hline
\end{tabular}

Table 2. The Knee Society Clinical Rating System before, Six and Eighteen months after Total Knee Arthroplasty ( $N=70)$

* Higher scores show better status.

** Comparison between six months after surgery and before surgery.

*** Comparison between eighteen months after surgery and six months after surgery.

\begin{tabular}{|c|c|c|}
\hline Complication & Frequency & Percent \\
\hline Infection & 7 & 10 \\
\hline Deep vein thrombosis & 3 & 4.3 \\
\hline Early loosening & 2 & 2.9 \\
\hline Dislocation & 1 & 1.4 \\
\hline Rubbing & 1 & 1.4 \\
\hline Nerve damage & 1 & 1.4 \\
\hline No complication & 55 & 78.6 \\
\hline
\end{tabular}

Table 3. Postoperative (Eighteen Months) Complications in Patients underwent Total Knee Arthroplasty $(n=70)$

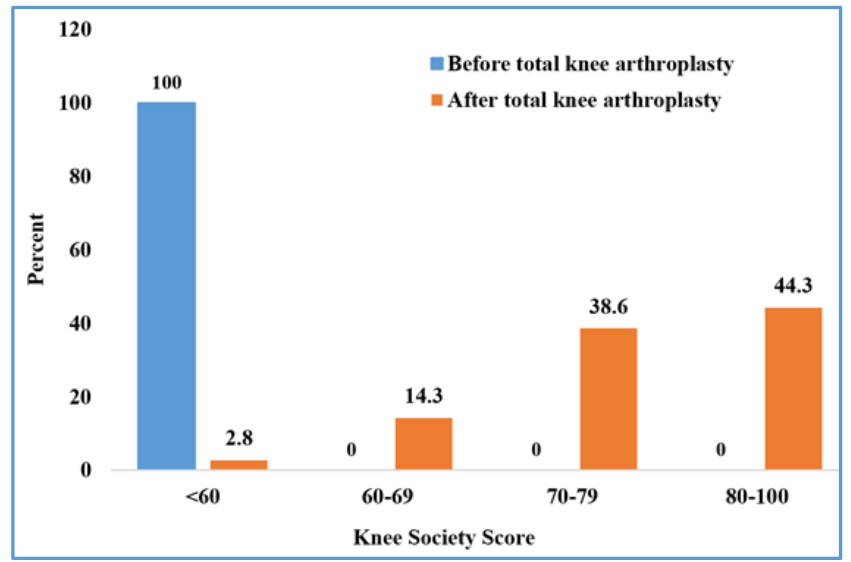

Figure 1. Distribution of Patients according to

Preoperative and Postoperative (eighteen months of surgery) Knee Society Score ( $=70)$. Higher Scores show better status (Excellent, 80-100; Good, 70-79; Fair, 60-69; Weak, <60).
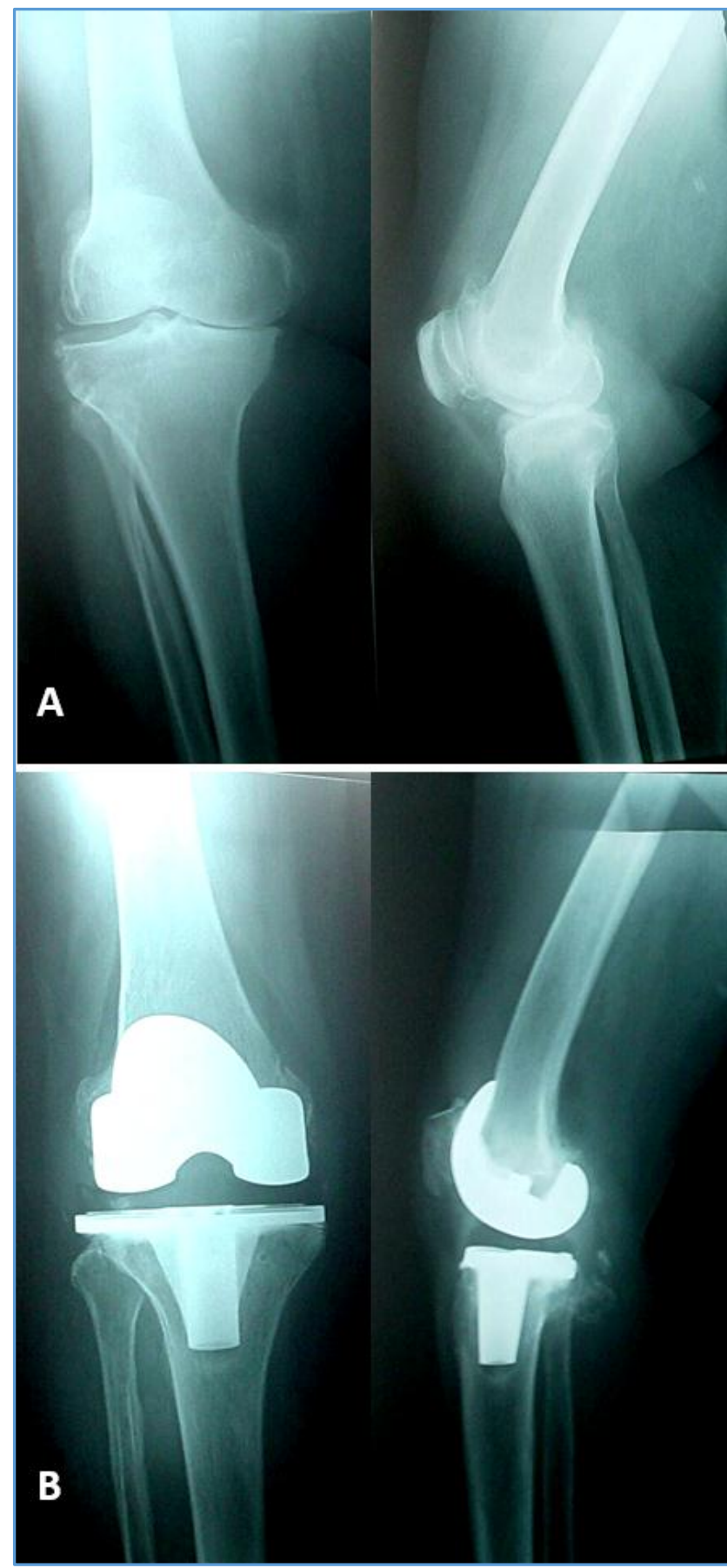

Figure 2. A) Patient with Severe Osteoarthritis of Right Knee before Total Knee Arthroplasty; B) Eighteen months after Total Knee Replacement, Femoral and Tibial components remained Stable

\section{DISCUSSION}

As observed in our study, total patients' functional score significantly increased over the eighteen months of follow-up. In their study, Dowsey et al(16) investigated the changes of patients' pain and function before and after total knee arthroplasty between Sweden and Australia. They found that rate of improvements was similar between the two countries and also they showed that total knee arthroplasty led to increase in function and decrease in pain.(16) With increase in life expectancy, rate of total knee arthroplasty is rising to improve quality of life and motility. In a study by Moussa et al.(17) They assessed outcome of use of stemmed constrained condylar knee implant in total knee arthroplasty. The authors 
stated that surgery outcome in group with stemmed constrained condylar knee implant was the same as in group without the given implant and totally total knee arthroplasty increased function and decreased pain and stiffness.(17) The key definition for complete improvement is not only right treatment, but also is restoring strength, coordination and range of motion of the joints. Glass et al(18) concluded that total knee arthroplasty causes changes in flexion and extension, leading to increase in range of joint motion.

In this study more than $80 \%$ of the patients had excellent or good final knee score (score $>70$ ), which was a very good outcome. On the other hand, in another study from Iran, which was conducted by Tahmasebi et al.(19) $53 \%$ of the subjects scored more than 70 . One of the reasons for the difference between their results and the present study can be related to pain management after the surgery, because they did not state anything about controlling postoperative pain. It is of importance that reducing postoperative pain has a considerable role in faster rehabilitation and achieving optimal range of motion of the patients and better outcomes. In other survey by Karimi Mobarakeh et al,(20) on 54 patients undergoing total knee arthroplasty, patients' function increased from 41 scores to 72 scores. In the present study, score of function increased significantly from 32 to 66 as well. In the research by Masoudifar et al,(21) score of range of motion increased over the first 4 weeks of surgery which was similar to our results.

The most important aim of treatment of such patients is lack of pain in rest and activity after the surgery. Lack of movement restrictions and independence in daily activities are next purposes. Jacobs et al(22) stated that total knee arthroplasty is associated with decrease in patients' pain and their satisfaction. Nguyen et al(23) mentioned that it is better to classify patients in terms of severity of pain and function. This classification leads to better evaluation of the surgery outcome. The authors also declared that the patients with severe pain and poor function achieve best outcome by comparison in other groups, patient's independence in daily activity may decrease postoperatively. The reduction in independence mostly results from limitation in patient's activities due to pain and fear of making problem in the foot underwent surgery and also because of decrease in motility of functional balance in the patient. Chronic pain can interact with patient's daily living activity and can lead to muscle weakness and consequently to decrease in range of joint motion. With decrease in joint motion, the muscles become weak and will no longer be able to protect the joint. $(24,25)$ Therefore, pain is one of the principle indications for total knee arthroplasty. In this research, score of patients' pain had a significant decrease over the postoperative follow-up period and they were satisfied.

Total knee arthroplasty can be associated with a number of complications, of which the most common is pain and range of motion limitation which can cause a considerable dysfunction postoperatively.(26,27) In the present study, more than half of the patients reported no complications. In other subjects, infection was the most frequent postoperative complication. In the study by Karimi Mobarakeh et al,(20) infection and delay in wound healing were seen in one and two subjects, respectively. They reported less number of complications in their patients.

\section{CONCLUSION}

The results of the present study showed that total knee arthroplasty could acceptably lead to improvement of knee function in the patients with knee arthropathy who underwent the surgery and could be correlated with patients' satisfaction. By further investigating advantages and disadvantages of total knee arthroplasty, we can help patients with degenerative diseases better to get rid of pain and dependence on others. It is suggested that further research be conducted with longer study period to assess long-term postoperative complications.

\section{ACKNOWLEDGEMENT}

The authors would like to thank the Vice Chancellor for the Research of Babol University of Medical Sciences for financial support. We are also thankful to Dr. Masoud Bahrami, Dr. Mani Falsafi and Dr. Rahmatollah Jokar for their advise. Besides, we thank Ms. Sekineh Kamali Ahangar, member of Clinical Research Development Centre of Shahid Beheshti Hospital, for her help in preparing the manuscript.

\section{REFERENCES}

[1] Carr AJ, Robertsson 0, Graves S, et al. Knee replacement. Lancet 2012;379(9823):1331-40.

[2] Sukumarapillai S, Indulekha $H$. The association between body mass index and duration of spinal anaesthesia in adults undergoing total knee replacement arthroplasty - an observational study. J Evolution Med Dent Sci 2017;6(32):2665-9.

[3] Katz JN. Editorial: appropriateness of total kneearthroplasty. Arthritis Rheumatol 2014;66(8):1979-81.

[4] Singh JA, Vessely MB, Harmsen WS, et al. A populationbased study of trends in the use of total hip and total knee arthroplasty, 1969-2008. Mayo Clin Proc 2010;85(10):898-904.

[5] National Joint Registry. National Joint Registry for England and Wales: 7th annual report. 2010. http://www.njrcentre.org.uk/NjrCentre/Portals/0/NJ R\%207th\%20Annual\%20Report\%202010.pdf (accessed Dec 16, 2011).

[6] Lohmander LS. Knee replacement for osteoarthritis: facts, hopes and fears. Medicographia 2013;35(2):1818.

[7] Lowe MCJ, Barker KL, Dewey M, et al. Effectiveness of physiotherapy exercise after knee arthroplasty for osteoarthritis: systematic review and meta-analysis of randomised controlled trials. BMJ 2007;335(7624):812.

[8] Parvez A, Gyaneshwar T, Amit S, et al. Functional outcome of posterior stablising total knee arthoplasty. J Evolution Med Dent Sci 2013;2(15):2417-25.

[9] Valle DC, Rosenberg A. Indications for total knee replacement. In: Callaghan J, Rosenberg A, Rubash $\mathrm{H}$, et al. eds. The adult knee. $1^{\text {st }}$ edn. Philadelphia, PA: Lippincott Williams \& Wilkins 2003: p. 1047-57.

[10] Healy WL, Valle DCJ, Iorio R, et al. Complications of total knee arthroplasty: standardized list and definitions of the Knee Society. Clin Orthop Relat Res 2013;471(1):215-20. 
[11] Hanjagi MY, Varkey P, Prakash V. Prospective study of efficacy of local infiltration analgesia and epidural analgesia in patients undergoing total knee replacements. J Evolution Med Dent Sci 2017;6(41):3214-8.

[12] Insall JN, Dorr LD, Scott RD, et al. Rationale of the Knee Society clinical rating system. Clin Orthop Relat Res 1989;(248):13-4.

[13] Rohra N, Suri HS, Gangrade K. Functional and radiological outcome of Schatzker type V and VI tibial plateau fracture treatment with dual plates with minimum 3 years follow-up: a prospective study. J Clin Diagn Res 2016;10(5):RC05-RC10.

[14] Bach CM, Nogler M, Steingruber IE, et al. Scoring systems in total knee arthroplasty. Clin Orthop Relat Res 2002;(399):184-96.

[15] Oishi K, Tsuda E, Yamamoto $Y$, et al. The Knee injury and Osteoarthritis Outcome Score reflects the severity of knee osteoarthritis better than the revised Knee Society Score in a general Japanese population. Knee 2016;23(1):35-42.

[16] Dowsey M, Robertsson O, Sundberg M, et al. Variations in pain and function before and after total knee arthroplasty: a comparison between Swedish and Australian cohorts. Osteoarthritis Cartilage 2017;25(6):885-91.

[17] Moussa ME, Lee YY, Patel AR, et al. Clinical outcomes following the use of constrained condylar knees in primary total knee arthroplasty. J Arthroplasty 2017;32(6):1869-73.

[18] Glass NA, Segal NA, Callaghan JJ, et al. Comparison of the extent to which total hip and total knee arthroplasty restore patient-reported physical function. Osteoarthritis Cartilage 2016;24(11):187582.
[19] Tahmasebi MN, Motaghi A, Shahrezaee M. Total knee arthroplasty in patients with osteoarthritis: results of 34 operations. Tehran Univ Med J 2009;67(2):146-50.

[20] Mobarakeh KM, Saeed A, Nemati A. Knee replacement (short-term results). IJOS 2007;5(4):165-70.

[21] Masoudifar M, Noorian N, Motieifar $M$, et al. Comparison of performance and pain intensity after total knee arthroplasty using general or regional anesthesia. Journal of Isfahan Medical School 2012;30(203):1313-20.

[22] Jacobs CA, Christensen CP, Karthikeyan T. Greater medial compartment forces during total knee arthroplasty associated with improved patient satisfaction and ability to navigate stairs. J Arthroplasty 2016;31(Suppl 9):87-90.

[23] Nguyen UD, Ayers DC, Li W, et al. Preoperative pain and function: profiles of patients selected for total knee arthroplasty. J Arthroplasty 2016;31(11):2402-7. e2.

[24] Lee YS. Comprehensive analysis of pain management after total knee arthroplasty. Knee Surg Relat Res 2017;29(2):80-6.

[25] Sakellariou VI, Poultsides LA, Ma Y, et al. Risk assessment for chronic pain and patient satisfaction after total knee arthroplasty. Orthopedics 2016;39(1):55-62.

[26] Belmont PJ Jr, Goodman GP, Waterman BR, et al. Thirty-day postoperative complications and mortality following total knee arthroplasty: incidence and risk factors among a national sample of 15, 321 patients. J Bone Joint Surg Am 2014;96(1):20-6.

[27] Ledford CK, Chalmers BP, Statz JM, et al. Primary total knee arthroplasty after solid organ transplant: survivorship and complications. J Arthroplasty 2017;32(1):101-5 